Mon. Not. R. Astron. Soc. 000, 11?? (2009) Printed 16 July $2019 \quad$ (MN LATEX style file v2.2)

\title{
Shock fronts in the long GRB031203 host galaxy
}

\author{
M. Contini \\ School of Physics and Astronomy, Tel Aviv University, Tel Aviv 69978, Israel
}

16 July 2019

\begin{abstract}
The detailed modelling of the spectra observed from the long GRB031203 host galaxy at different epochs during the 2003-2009 years is presented. The line profiles show FWHM of $\sim 100 \mathrm{~km} \mathrm{~s}^{-1}$. A broad line profile with FWHM $\leqslant 400 \mathrm{~km} \mathrm{~s}^{-1}$ appears in the line sockets from the 2009 observations. We suggest that the narrow lines show the velocity of star-burst (SB) debris, while the broad ones are due to the wind from SB stars. The spectra are emitted from the gas downstream of different shock fronts which are at work on the edges of the emitting clouds. A head-on-back shock appears when the wind from the SB stars reaches the internal edge of the SB debris moving outwards. A head-on shock is created by collision of the debris with the ISM clouds. Line ratios in both cases are calculated by the coupled effect of shock and photoionization from the SB. The models selected by fitting the calculated to the observed line ratios show that the ionization parameters, the shock velocities and the gas pre-shock densities slowly decrease with time. Oxygen metallicities $(12+\log (\mathrm{O} / \mathrm{H})=8.3-8.48)$ are lower than solar (8.82) by a factor $<3$ and nitrogen metallicities are lower than solar $(12+\log (\mathrm{N} / \mathrm{H})=8.0)$ by factors of $3-5$.
\end{abstract}

Key words: radiation mechanisms: general — shock waves - galaxies: GRB host — galaxies: high redshift

\section{INTRODUCTION}

'Long duration gamma-ray bursts (LGRB) and short dura' tion GRB (SGRB) have different origins. LGRB derive from implosion of a massive star while SGRB are the product , of (neutron star) NS-NS or NS-BH (black hole) collisions. LGRBs appear among young population stars (Fruchter et al 2006, Savaglio et al 2009, etc), while for SGRBs both young and old population stars were found (e.g. Berger 2009, Savaglio et al. 2009, etc.). GRB are distinguished by time periods, which are evident from the distribution of the data. The burst period is $T_{90} \leqslant 2$ s for SGRB, where $T_{90}$ is the time interval between 5 percent to 95 percent gamma-ray photons collected by a given instrument (Kouveliotou et al 1993). LGRB have longer duration times, up to several minutes (Kouveliotou et al). Fig. 1 shows the dicotomy of the GRB phenomenon. GRB031203 triggered the imager onBoard the Integral Satellite (IBIS) instrument of the international gamma-ray astrophysics laboratory (INTEGRAL) on 2003 December 3 (Gotz et al 2003) with a duration of $\sim 30 \mathrm{~s}$ and a peak flux of $1.310^{-7} \mathrm{erg} \mathrm{cm}^{-2} \mathrm{~s}^{-1}(20-200 \mathrm{keV}$, Meneghetti \& Gots 2003). A compact dwarf galaxy coinciding with the X-ray source (Hsia et al 2003) was later identified as the GRB031203 host at $\mathrm{z}=0.1055$ (Prochaska et al 2004). A SN event led to the discovery of supernova SN2003lw (Tagliaferri et al 2004). The X-ray and radio af- terglows were soon discovered at European Southern Observatory (ESO), starting near infrared (NIR) observations at the New Technology Telescope (NTT) 7 hours after the GRB and then for the X-ray source in GRB031203. Guseva et al (2011) reported the general claim that "there are no evidences that host galaxies are not peculiar and are similar to star-forming galaxies in the local and distant universe". They concluded that for galaxies at $z \geqslant 0.1$, the properties of the LGRB environment can be retrieved from the ISM of the entire galaxy as occurs for GRB031203. The afterglow of GRB031203 was very weak, the faintest ever detected in the optical/NIR. The detection of the SN optical light implies that an extreme dust obscuration was not the reason for such faintness.

In the latest years we have been investigating LGRB and SGRB host galaxy spectra by the detailed calculation of the physical conditions and the element abundances. The results suggest that LGRB and SGRB host galaxies may be distinguished by the $\mathrm{N} / \mathrm{O}$ distribution trend as function of the redshift at low $\mathrm{z}(\mathrm{z}<4$ Contini 2017). Moreover, it seems that in some of the SGRB hosts (e.g. SGRB100628A) an active galactic nucleus (AGN) can be present and even coupled to the star-burst (Contini 2019). In this paper we calculate by detailed modelling the line and continuum spectra of LGRB031203 host galaxy. Observations at different epochs were presented by Prochaska et al (2004), Margutti et al 




Figure 1. Distribution of GRB redshifts versus t90. Open squares: SGRB; filled squares: LGRB. Data from Wei et al (2014).

(2007), Guseva et al (2011), Watson et al (2011), etc. Observations of the spectra by Margutti et al (2007) at different times in the years 2003-2004 provide the opportunity to follow the variations of the emitted line ratios observed from GRB031203 host on a short time scale. We focus on line and continuum modelling throughout the host galaxy, in particular on the physical conditions and metallicities, on the contribution to the continuum SED of gas bremsstrahlung and reradiation by dust, on the contribution of the underlying old star population in the IR and of radio synchrotron emission. To calculate the physical conditions and the element abundances in LGRB031203 host we use composite models which account for the photoionizing flux from the star-burst (SB) coupled with shocks.

Afterglow issues, host physical features and metallicities which characterize GRB031203 are summarized in Sect. 2. The calculations are presented in Sect. 3 and the results of modelling the line ratios observed by Prochaska et al, Margutti et al (2007), Guseva et al (2011) and Watson et al (2011) appear in Sect. 4. In Sect. 5 the continuum SED observations are compared with the results of the models that were adopted to fit the line ratios. Concluding remarks follow in Sect. 6.

\section{GENERAL GRB FEATURES}

In the following we summarize some of the GRB issues which are significant to GRB031203, in particular, a weak afterglow, a complex hydrodynamic host field and low metallicities.

\subsection{Afterglow}

An afterglow arises when the GRB ejecta are slowed down by collision with circum-burst matter. It shows lower energy than the GRB, long lasting X-ray, optical emission and a long-lived radio emission due to a blast wave subrelativistic envelope. The overall observed isotropic luminosity is $\sim$
$10^{51}-10^{52} \mathrm{erg} \mathrm{s}^{-1}$ (Piran 2004). Berger (2009) by the observational evidence suggests a link of LGRB with the end of life of massive stars (Woosley 1993, Woosley \& Bloom 2006) from their association with core collapse supernovae (Hjorth et al 2003). As a consequence the fireball shell expands (Kobayashi \& Zhang 2003) into the pre-burst stellar wind of the progenitor star. The wind has a density distribution $\rho \propto \mathrm{R}^{-2}$. The interaction between the shell and the wind leads to two shocks: one propagating in reverse into the shell and the other propagating forward into the wind. The velocity of the shock propagating in reverse towards the burst decreases due to high density in the ejecta. This could explain the short lifetimes of some afterglows. Giannios et al (2008) claim that the early optical afterglow light curves depend critically on the existence of a reverse shock into a strong magnetized ejecta. In the first afterglow period the reverse shock plays a main role or disappears due to a strong magnetic field (Giannios et al 2008, Zhang \& Kobayashi 2005, Sari \& Piran 1999, etc). A reverse shock may also dominate the emission in later afterglow phases. After the reverse shock reaches the back part of the ejecta, there is an initial phase of interaction where shocks and rarefaction waves cross the ejecta. Then the whole structure relaxes to a self-similar solution. The X-ray energy emitted during the afterglow is relatively high (e.g. $2-10 \mathrm{keV}$ at 11 hours after GRB, Piran 2004) and is most probably emitted during free expansion. Optical afterglows disappear after a few months, therefore they do not prevent to search for GRB host galaxies (Chen 2018). Recombination coefficients downstream of the shock fronts determine the lifetime of the afterglow. The recombination lifetime of hydrogen ions is $\mathrm{t}_{\text {rec }} \sim 1 /\left(\mathrm{n} \alpha_{R}\right)$, where $\alpha_{R}=4 \times 10^{-13}\left(\mathrm{~T} / 10^{4}\right)^{-0.72}$ is the recombination coefficient of $\mathrm{H}$ (Aldrovandi \& Péquignot 1973). A very high density $n$ (adapted to the ejecta) can reduce $t_{r e c}$ to the order of seconds. In the low density case, emission features from the afterglow can be seen even after its disappearance. The afterglow is characterised by synchrotron radiation observable from several days to a few months (Chen 2018). After the first afterglow period and an initial stage of expansion in a dilute ISM, the ejecta will interact with a higher density medium in the host galaxy.

\subsection{Host galaxy radiation and collisional ionization sources}

A SB is generally present in the GRB host galaxy. The full width at half maximum (FWHM) of the GRB031203 line profiles from Guseva et al (2011, fig. 7) indicates a complex kinetic field with high and low velocities. The narrow line velocity range is $\sim 90-130 \mathrm{~km} \mathrm{~s}^{-1}$ while the broad line one is $\sim 200-330 \mathrm{~km} \mathrm{~s}^{-1}$. This suggests that composite models which account for the photoionization flux from the SB and for shocks, could be appropriate and could add some information about the physical conditions in the host galaxy. The wind from the SB stars affects the host galaxy clouds. The gas is ionized to high levels by radiation and collisional process. Recombination times depend on the physical conditions in the surrounding medium and within the host galaxy. Temperatures can reach $\mathrm{T}>10^{6} \mathrm{~K}$. The expanding shock propagates outwards throughout the circumstellar medium. The evolution of the emitted spectra (line and continuum) observed from the host galaxy is smooth or disturbed depend- 


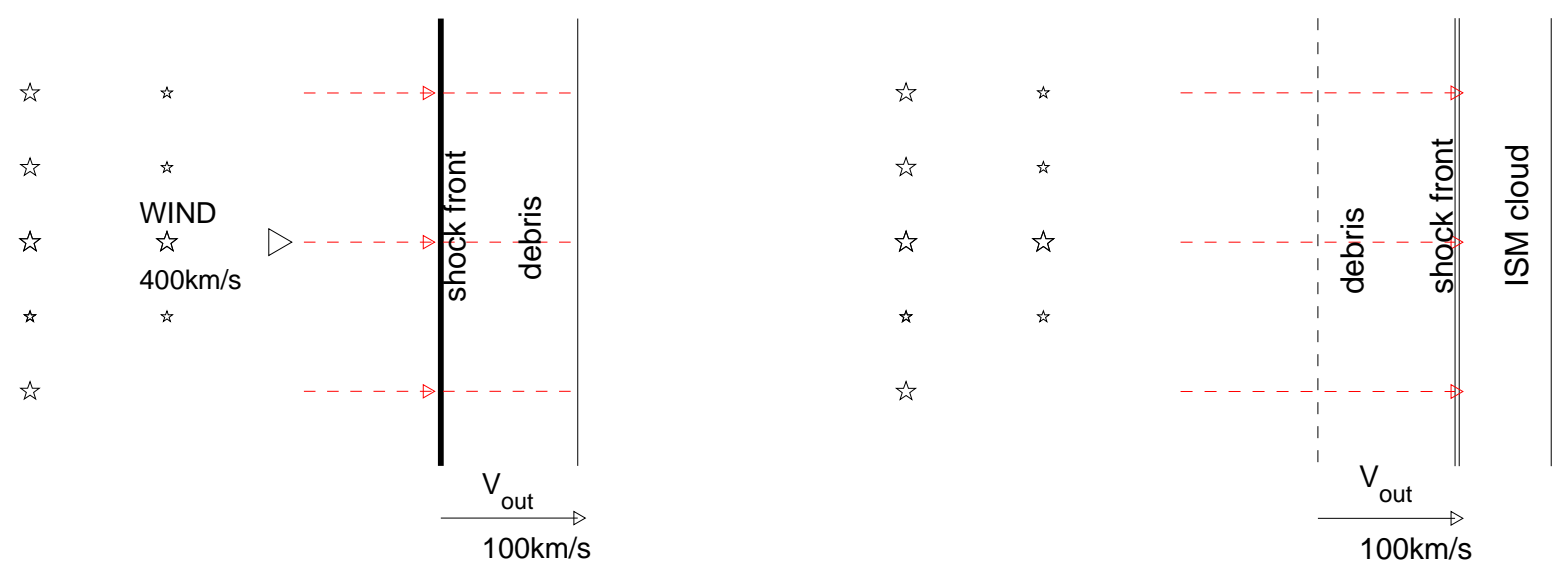

Figure 2. Sketch of the head-on (right) and head-on-back (left) shock fronts. Red dotted lines represent the radiation flux from the SB.

ing on the ISM physical conditions throughout the host. After a certain time, the direct effect of the burst on the emitting gaseous and dusty clouds may disappear.

\subsection{Metallicities}

In GRB hosts, star formation and star formation rates (SFR) are at most calculated from the $\mathrm{H} \alpha$ line and therefore they depend on the emitting gas conditions such as the density and the temperature. Perley et al (2016) investigating whether the GRB is affecting the SFR independently from other factors, concluded that the most "invoked possibility" is a dependence on metallicity. GRBs affect the host galaxy properties in the surrounding medium. Therefore, the heavy elements cannot be neglected. SFR is proportional to the fractional abundance of the different ions in different ionization levels. A low metal abundance "both discourages mass loss from the parent stars and encourage mixing". Therefore, Perley et al predicted that GRB should occur in very metal poor environments. Single star models (MacFadyen \& Woosley 1999, Maeder et al 2005, Hitschi et al 2005, etc ) imply that GRBs should occur mainly in very metal poor environments. On the other hand, to produce GRB through an energetic jet, a high metallicity is needed, because a higher metal abundance produces stronger stellar winds, greater mass loss and less interior mixing (Crowther et al 2002, Heger et al 2003, Vink \& de Koter 2005, Hirschi et al 2008). How much does the progenitor's low metallicity affect the metallicity of the host clouds depends also on the merging processes. The metallicity upper limit to GRB production is estimated by Wolf \& Podsidlowski (2007) to $12+\log (\mathrm{O} / \mathrm{H})<8.7$. Chen $(2018)$ reconciled the GRB origin from the death of rapid evolving massive stars with active forming regions into the epoch of reionization.

\section{CALCULATIONS}

\subsection{Description of the code}

We use composite models which account consistently for photoionization and shocks. The code sumA (see also Fer- land et al 2016) is adopted. The main input parameters are those which are used for the calculations of the line and continuum fluxes. They account for photoionization and heating by primary and secondary radiation and collisional process due to shocks. The input parameters such as the shock velocity $\mathrm{V}_{\mathrm{s}}$, the atomic preshock density $\mathrm{n}_{0}$ and the preshock magnetic field $\mathrm{B}_{0}$ (for all models $\mathrm{B}_{0}=10^{-4}$ Gauss is adopted) define the hydrodynamical field. They are used in the calculations of the Rankine-Hugoniot equations at the shock front and downstream. They are combined in the compression equation (Cox 1972) which is resolved throughout each slab of the gas in order to obtain the density profile downstream. Primary radiation for SB in the GRB host galaxies is approximated by a black-body (bb). The input parameters that represent the primary radiation from the SB are the effective temperature $\mathrm{T}_{*}$ and the ionization parameter $U$. A pure blackbody radiation referring to $\mathrm{T}_{*}$ is a poor approximation for a star burst, even adopting a dominant spectral type (see Rigby \& Rieke 2004). However, it is the most suitable because the line ratios that are used to indicate $\mathrm{T}_{*}$ also depend on metallicity, electron temperature, density, ionization parameter, morphology of the ionized clouds and, in particular, the hydrodynamical picture. For an AGN, the primary radiation is the power-law radiation flux from the active centre $F$ in number of photons $\mathrm{cm}^{-2} \mathrm{~s}^{-1} \mathrm{eV}^{-1}$ at the Lyman limit and spectral indices $\alpha_{U V}=-1.5$ and $\alpha_{X}=-0.7$. The primary radiation source does not depend on the host physical condition but it affects the surrounding gas. This region is not considered as a unique cloud, but as a sequence of slabs with different thickness calculated automatically following the temperature gradient. The secondary diffuse radiation is emitted from the slabs of gas heated by the radiation flux reaching the gas and by the shock. Primary and secondary radiation are calculated by radiation transfer.

In our model the line and continuum emitting regions throughout the galaxy cover an ensemble of fragmented clouds. The geometrical thickness of the clouds is an input parameter of the code $(D)$ which is calculated consistently with the physical conditions and element abundances of the emitting gas. The fractional abundances of the ions are calculated resolving the ionization equations for each element 
( $\mathrm{H}, \mathrm{He}, \mathrm{C}, \mathrm{N}, \mathrm{O}, \mathrm{Ne}, \mathrm{Mg}, \mathrm{Si}, \mathrm{S}, \mathrm{Ar}, \mathrm{Cl}, \mathrm{Fe}$ ) in each ionization level. Then, the calculated line ratios, integrated throughout the cloud thickness, are compared with the observed ones. The calculation process is repeated changing the input parameters until the observed data are reproduced by the model results, at maximum within 10-20 percent for the strongest line ratios and within 50 percent for the weakest ones.

However, some parameters regarding the continuum $\mathrm{SED}$, such as the dust-to-gas ratio $d / g$ and the dust grain radius $\mathrm{a}_{g r}$ are not directly constrained by fitting the line ratios. Dust grains are heated by the primary radiation and by mutual collision with atoms. The intensity of dust reprocessed radiation in the infrared (IR) depends on $d / g$ and $\mathrm{a}_{g r}$. In this work we use $d / g=10^{-14}$ by number for all the models which corresponds to $4.110^{-4}$ by mass for silicates (Draine \& Lee 1994). The distribution of the grain size along the cloud starting from an initial radius is automatically derived by SUMA, which calculates sputtering of the grains in the different zones downstream of the shock. The sputtering rate depends on the gas temperature, which is $\propto \mathrm{V}_{s}^{2}$ in the immediate post-shock region. In the high-velocity case $\left(\mathrm{V}_{\mathrm{s}} \geqslant\right.$ $500 \mathrm{~km} \mathrm{~s}^{-1}$ ) the sputtering rate is so high that the grains with $\mathrm{a}_{g r} \leqslant 0.1 \mu \mathrm{m}$ are rapidly destroyed downstream. So, only grains with large radius $\left(\mathrm{a}_{g r} \geqslant 0.1 \mu \mathrm{m}\right)$ will survive. On the other hand, the grains survive downstream of low-velocity shocks $\left(<200 \mathrm{~km} \mathrm{~s}^{-1}\right)$. Graphite grains are more sputtered than silicate grains for $\mathrm{T}=10^{6} \mathrm{~K}$ (Draine \& Salpeter 1979). Small grains (e.g. PAH) survive in the extended galactic regions on scales of hundred parsecs and lead to the characteristic features that appear in the SED. In conclusion, cold dust or cirrus emission results from heating by the interstellar radiation field, warm dust is associated with star formation regions and hot dust appears around AGN (Helou 1986) and in high velocity shock regimes. Therefore, we will consider relatively large grains, e.g. silicate grains with an initial radius of $0.1-1.0 \mu \mathrm{m}$

In the radio range the power-law spectrum of synchrotron radiation created by the Fermi mechanism at the shock front is seen in most galaxies. It is calculated by SUMA adopting a spectral index of -0.75 (Bell 1977).

\subsection{Calculation details}

The calculations initiate at the shock front where the gas is compressed and adiabatically thermalised, reaching a maximum temperature in the immediate post-shock region $\mathrm{T} \sim$ $1.5 \times 10^{5}\left(\mathrm{~V}_{\mathrm{s}} / 100 \mathrm{~km} \mathrm{~s}^{-1}\right)^{2}$. $\mathrm{T}$ decreases downstream following recombination. The cooling rate is calculated in each slab. The downstream region is cut into a maximum of 300 plane-parallel slabs with different geometrical widths calculated automatically, to account for the temperature gradient. In each slab, compression is calculated by the RankineHugoniot equations for the conservation of mass, momentum and energy throughout the shock front. Compression $\left(\mathrm{n} / \mathrm{n}_{0}\right)$ downstream ranges between 4 (the adiabatic jump) and $\geqslant 10$, depending on $V_{s}$ and $B_{0}$. The stronger the magnetic field, the lower the compression downstream, while a higher shock velocity corresponds to a higher compression. The ionizing radiation from an external source is characterised by its spectrum and by the flux intensity. The flux is calculated at 440 energies from a few $\mathrm{eV}$ to $\mathrm{keV}$. Owing to radiative transfer, the spectrum changes throughout the downstream slabs, each of them contributing to the optical depth. In addition to the radiation from the primary source, the effect of the diffuse radiation created by the gas line and continuum emission is also taken into account, using 240 energies to calculate the spectrum. For each slab of gas, the fractional abundance of the ions of each chemical element is obtained by solving the ionization equations $\mathrm{X}_{i+1} / \mathrm{X}_{i}=\mathrm{X}_{I}+\mathrm{X}_{I I}+\mathrm{X}_{I I I}$ (Contini \& Shaviv 1980), where $\mathrm{X}_{I}$ represents the contribution to ionization from the primary source (stars or AGN) (Williams 1973), $\mathrm{X}_{I I}$ represents the contribution of collisional ionization (Cox \& Tucker 1969) and $\mathrm{X}_{I I I}$ is the contribution of secondary radiation (Williams 1967). The parameters are described by Contini \& Aldrovandi (1983 and references therein) and Viegas-Aldrovandi \& Contini (1989 and references therein).

These equations account for the ionization mechanisms (photoionization by the primary and diffuse radiation, and collisional ionization) and recombination mechanisms (radiative, dielectronic recombinations), as well as charge transfer effects. The ionization equations are coupled to the energy equation if collision processes dominate, and to the thermal balance if radiative processes dominate. The latter balances the heating of the gas due to the primary and diffuse radiations reaching the slab with the cooling due to recombinations and collisional excitation of the ions followed by line emission, dust collisional ionization and thermal bremsstrahlung. The coupled equations are solved for each slab, providing the physical conditions necessary for calculating the slab optical depth, as well as its line and continuum emissions. The slab contributions are integrated throughout the cloud. In particular, the absolute line fluxes corresponding to the ionization level i of element $\mathrm{K}$ are calculated by the term $\mathrm{nK}(\mathrm{i})$, which represents the density of the ion $\mathrm{i}$. We consider that $\mathrm{nK}(\mathrm{i})=\mathrm{X}(\mathrm{i})[\mathrm{K} / \mathrm{H}] \mathrm{n}_{H}$, where $\mathrm{X}(\mathrm{i})$ is the fractional abundance of the ion $\mathrm{i}$ calculated by the ionization equations, $[\mathrm{K} / \mathrm{H}]$ is the relative abundance of the element $\mathrm{K}$ to $\mathrm{H}$ and $\mathrm{n}_{H}$ is the density of $\mathrm{H}$ (in number $\mathrm{cm}^{-3}$ ). In models including shock, $\mathrm{n}_{H}$ is calculated by the compression equation (Cox 1972) in each slab downstream. Accordingly, the abundances of the elements are given relative to $\mathrm{H}$ as input parameters.

Dust grains are coupled to the gas across the shock front by the magnetic field (Viegas \& Contini 1994). They are heated by radiation from the AGN and collisionally by the gas to a maximum temperature, which is a function of the shock velocity, of the chemical composition and of the radius of the grains, up to the evaporation temperature ( $\mathrm{T}$ (dust) $\geqslant 1500 \mathrm{~K})$. The grain radius distribution downstream is determined by sputtering, which depends on the shock velocity and on the density. Throughout shock fronts and downstream, the grains might be destroyed by sputtering.

Summarizing, the code starts by adopting an initial $\mathrm{T}_{\mathrm{e}}\left(10^{4} \mathrm{~K}\right)$ and the input parameters for the first slab. It then calculates the density from the compression equation, the fractional abundances of the ions from each level for each element, line emission, free-free emission and freebound emission. It re-calculates $\mathrm{T}_{\mathrm{e}}$ by thermal balancing or the enthalpy equation, and calculates the optical depth of the slab and the primary and secondary fluxes. Finally, it adopts the parameters found in slab i as initial conditions for slab $i+1$. Line and continuum intensities are integrated 
accounting for all the slabs. The number of lines calculated by SUMA is $>200$ for each model. They are calculated at the gaseous nebula that emits the spectrum, while the data are observed at Earth. Therefore they diverge by a factor $\left(\mathrm{r}^{2} / \mathrm{d}^{2}\right)$ that depends on the distance of the nebula from the radiation centre (r), and on the distance (d) of the galaxy to Earth. We then calculate the line ratios to a specific line (in the present case $\mathrm{H} \beta$, which is a strong line), and compare them with the observed line ratios.

On this basis we calculate a grid of models. The set of models (e.g. Table 1) which best reproduce the line ratios is selected. We obtain the final model by cross-checking the fit of the calculated continuum SED to the observed one.

\subsection{Modelling the continuum SED}

The models constrained by the line spectra give a hint about the relative importance of the different ionization and heating mechanisms which are recognised throughout the continuum SED in each of the objects.

The gas ionized by the $\mathrm{SB}$ (or AGN) radiation flux emits continuum radiation (as well as the line fluxes) from radio to $\mathrm{X}$-ray. The continuum accounts for free-free and free-bound radiation (hereafter addressed to as bremsstrahlung). The bremsstrahlung at $\nu<10^{14} \mathrm{~Hz}$ has a similar slope in all the diagrams. In fact, the bremsstrahlung continuum, emitted by free electrons accelerated in Coulomb collisions with positive ions (mostly $\mathrm{H}^{+}, \mathrm{He}^{+}$and $\mathrm{He}^{++}$) in nebulae of charge $\mathrm{Z}$ has an emission coefficient (Osterbrock 1974):

$$
\mathrm{J}_{\nu} \propto \mathrm{N}_{\mathrm{e}} \mathrm{N}_{+} \mathrm{Z}^{2}(\pi h \nu / 3 \mathrm{kT})^{1 / 2} \mathrm{e}^{-(h \nu / k T)}(1)
$$

The photoionization radiation flux can heat the gas to $\mathrm{T} \sim$ $2-4 \times 10^{4} \mathrm{~K}$, while the gas is heated collisionally by the shock to a maximum of $\mathrm{T} \propto\left(\mathrm{V}_{\mathrm{s}}\right)^{2}$, where $\mathrm{V}_{\mathrm{s}}$ is the shock velocity. The cooling rate downstream depends on $\mathrm{N}_{\mathrm{e}} \mathrm{N}_{+}\left(\mathrm{N}_{+}\right.$is the proton density). The trend of the bremsstrahlung as function of $\nu$ follows the interplay between $\mathrm{T}$ and $\nu$. High temperatures of the emitting gas determine the bremsstrahlung maximum at high $\nu$. At $\mathrm{T} \sim 1-4 \times 10^{4} \mathrm{~K}$ the exponential term is significant at frequencies between $10^{14}$ and $10^{15} \mathrm{~Hz}$. The temperatures are calculated by thermal balancing between the heating rates which depend on the photoionizing flux and the cooling rates by free-free, free-bound and line emission. Therefore, the radiation effect is seen mainly in this frequency range. In the radio range, the exponent in eq (1) tends to 0 and the continuum is $\propto \nu^{1 / 2}$. So the SEDs in all the diagrams of all galaxy types have similar trends at relatively low frequencies and the dust reprocessed radiation bump in the IR is clearly recognizable.

In conclusions, i) the black body radiation corresponding directly to the temperature dominating in the star-burst is seldom observed in the UV, because absorption is very strong in this frequency range due to strong line formation. ii) The shock effect throughout the SED can be recognized from the maximum frequency and intensity of the dust reprocessed radiation peak in the infrared and of the bremsstrahlung at high frequencies. iii) The gas ionized by the $\mathrm{SB}$ (or AGN) radiation flux emits bremsstrahlung from radio to $\mathrm{X}$-ray. The black body emission from the background old star population with $\mathrm{T}_{b b} \sim 3000-8000 \mathrm{~K}$ generally emerges over the bremsstrahlung throughout the SED in the near-IR(NIR) - optical range. iv) In the radio range synchrotron radiation created by the Fermi mechanism is recognized by its spectral index. Thermal bremsstrahlung in the radio range has a steeper trend which becomes even steeper by self-absorption at low $\nu$. In the far-IR only comparison with the observation data indicates the source of the continuum radiation flux, because thermal bremsstrahlung, synchrotron radio and cold dust reradiation may be blended.

\section{MODELLING GRB031203 LINE SPECTRA}

\subsection{Spectral characteristics}

In the following we present the results obtained by the detailed modelling of the LGRB031203 host galaxy spectra observed by Prochaska et al (2004) on the Magellan/Baade $6.5 \mathrm{~m}$ telescope and Inamori Magellan Areal Camera and Spectrograph (IMACS), at different epochs (2003-2004) by Margutti et al (2007) on the ESO-Very Large Talescope (VLT) focal reducer/low-resolution spectrograph (FORS) and by Guseva et al (2011) by the VLT X-shooter. Both narrow and broad lines were observed at later times by $\mathrm{Gu}$ seva et al, in March 2009. Watson et al (2011) presented the first spectroscopic study of the LGRB031203 host galaxy in the mid-IR (5-40 $\mu \mathrm{m})$ by VLT X-shooter on 2009 March 17 and Spitzer photometric observations from the UV -mid-IR (0.35-40 $\mu \mathrm{m})$ with detections and upper limits at far-IR, submillimeter and radio wavelengths. They were carried out on 2005 May 28. Observation epochs and $\mathrm{H} \beta$ observed fluxes are summarised in Table 1.

The sketch in Fig. 2 (right panel) represents the clouds emitting the narrow lines (models mod0-mod6 in Tables 24 ), while the left panel explains the broad lines emission (model mod7 in Tables 3 and 4). Both strong and weak shock fronts are reached by the photoionization flux from the $\mathrm{SB}$. In the case of a SB, the clouds (representing the debris from the $\mathrm{SB}$ ) propagate outwards with velocity $\mathrm{V}_{\text {debris }} \sim 100$ $\mathrm{km} \mathrm{s}^{-1}$ from the radiation source i.e. from the SB. The SB debris collide with the ISM clouds which are nearly standing with velocities $\mathrm{V}_{I S M} \sim 30 \mathrm{~km} \mathrm{~s}^{-1}$. A head-on shock follows with $\mathrm{V}_{\mathrm{s}}=\mathrm{V}_{\text {debris }}+\mathrm{V}_{\text {ISM }} \sim 100-130 \mathrm{~km} \mathrm{~s}^{-1}$. Moreover, a relatively high velocity wind $\left(\mathrm{V}_{\text {wind }} \sim 400 \mathrm{~km} \mathrm{~s}^{-1}\right)$ originating from the SB stars reaches the expanding debris yielding a head-on-back shock with $\mathrm{V}_{\mathrm{s}}=\mathrm{V}_{\text {wind }}-\mathrm{V}_{\text {debris }} \sim 300 \mathrm{~km} \mathrm{~s}^{-1}$. A high velocity wind at earlier times $\left(\sim 1000 \mathrm{~km} \mathrm{~s}^{-1}\right)$ could have easily destroyed the gaseous clouds by collisions. It was shown by Schiano $(1985,1986)$ that clouds with preshock densities $<100 \mathrm{~cm}^{-3}$ are swept from the region of free flowing winds (Contini \& Viegas-Aldrovandi 1990). Regarding line profiles in wind regimes, although in a different contest, Vogel \& Nussbaumer (1994) noticed that the luminosity of the hot component of the symbiotic star AG Peg decreased from $1600 \mathrm{~L}_{\odot}$ in 1978 to $500 \mathrm{~L}_{\odot}$ in 1990 and furthermore to $400 \mathrm{~L}_{\odot}$ in 1993 . NV and HeII lines exhibited a pure wind profile on top of which a nebular contribution appeared in 1981 and 1986, respectively. These profiles were recognised by Penston \& Allen (1985) as a convolution of a nebular component and a broad line component, which they attributed to a wind lost by the hot companion with a velocity of $\sim 900$ $\mathrm{kms}^{-1}$. In all the observations the wind velocity retained this value, although the strength gradually decreased. This decline was followed by qualitative changes in the profiles of the emission features. 
Table 1. Observation details (2003-2009)

\begin{tabular}{lccccccc}
\hline \hline & obs $^{0}$ & obs $^{1}$ & obs $^{2}$ & obs $^{3}$ & obs $^{4}$ & obs $^{5}$ & obs $^{6}$ \\
day & 2003 Dec 6.3 & 2003 Dec 20 & 2003 Dec 30 & 2004 Mar 2 & 2004 Sep 2 & 2009 Mar 17 & 2009 Mar 17 \\
H $\beta^{7}$ & $51.8 \pm 0.8$ & $2142.39 \pm 326.70$ & $2135.91 \pm 10$ & $1932.24 \pm 571.15$ & $1949.33 \pm 263.49$ & $3.85 \pm 0.04$ & - \\
Telescope & Magellan/Baade & ESO-VLT & ESO-VLT & ESO-VLT & ESO-VLT & VLT & VLT \\
& IMACS & FORS2 & FORS1 & FORS1 & FORS1 & X-shooter & X-shooter \\
\hline
\end{tabular}

${ }^{0}$ Prochaska et al (2004); ${ }^{1}$ Margutti et al (2007); ${ }^{2}$ Margutti et al (2007); ${ }^{3}$ Margutti et al (2007); ${ }^{4}$ Margutti et al (2007); ${ }^{5}$ Guseva et al (2011); ${ }^{6}$ Watson et al (2011); ${ }^{7}$ in $10^{-16} \mathrm{erg} \mathrm{cm}^{-2} \mathrm{~s}^{-1}$ observed at Earth

Table 2. Extinction corrected line ratios to $\mathrm{H} \beta$ at different epochs from Prochaska et al (2004) and Margutti et al (2007)

\begin{tabular}{lcccccccccc}
\hline \hline & obs $^{0}$ & $\bmod 0$ & obs $^{1}$ & $\bmod 1$ & obs $^{2}$ & $\bmod 2$ & obs $^{3}$ & $\bmod 3$ & obs $^{4}$ & $\bmod 4$ \\
\hline$[\mathrm{OII}] 3728.8$ & 1.06 & 1.06 & 1.20 & 1.26 & 1.33 & 1.33 & 1.20 & 1.23 & 1.11 & 1.10 \\
{$[\mathrm{NeIII}] 3868+$} & 0.83 & 0.90 & 0.98 & 1.00 & 0.88 & 0.97 & 0.84 & 1. & 0.83 & 1.00 \\
$\mathrm{H} \gamma$ & 0.49 & 0.46 & 0.46 & 0.46 & 0.45 & 0.46 & 0.43 & 0.46 & 0.45 & 0.46 \\
{$[\mathrm{OIII}] 4363$} & 0.11 & 0.08 & 0.08 & 0.08 & 0.08 & 0.08 & 0.07 & 0.08 & 0.08 & 0.08 \\
$\mathrm{HeI} 4471.5$ & 0.05 & 0.05 & 0.03 & 0.05 & - & - & - & - & - & - \\
$\mathrm{H} \beta$ & 1 & 1 & 1 & 1 & 1 & 1 & 1 & 1 & 1 & 1 \\
{$[\mathrm{OIII}] 5007+$} & 8.46 & 8.40 & 8.72 & 8.50 & 8.52 & 8.69 & 8.61 & 8.55 & 8.53 & 8.50 \\
$\mathrm{HeI} 5876$ & 0.12 & 0.14 & 0.11 & 0.14 & 0.12 & 0.14 & 0.12 & 0.14 & 0.14 & 0.14 \\
{$[\mathrm{OI}] 6300+$} & 0.02 & 0.01 & 0.04 & 0.02 & 0.03 & 0.016 & 0.04 & 0.01 & 0.03 & 0.01 \\
{$[\mathrm{SIII}] 6312$} & 0.02 & 0.02 & 0.02 & 0.02 & 0.02 & 0.02 & 0.02 & 0.02 & 0.01 & 0.02 \\
{$[\mathrm{NII}] 6548+$} & 0.21 & 0.21 & 0.21 & 0.25 & 0.19 & 0.20 & 0.18 & 0.20 & 0.19 & 0.18 \\
$\mathrm{H} \alpha$ & 2.82 & 2.90 & 2.83 & 2.9 & 2.78 & 2.91 & 2.79 & 2.89 & 2.96 & 2.89 \\
{$[\mathrm{SII}] 6717$} & 0.08 & 0.08 & 0.09 & 0.08 & 0.10 & 0.08 & 0.10 & 0.1 & 0.10 & 0.10 \\
{$[\mathrm{SII}] 6731$} & 0.07 & 0.09 & 0.07 & 0.08 & 0.07 & 0.08 & 0.08 & 0.08 & 0.08 & 0.08 \\
{$[\mathrm{AIII}] 7136$} & 0.07 & 0.06 & 0.07 & 0.10 & 0.07 & 0.07 & 0.07 & 0.1 & 0.06 & 0.10 \\
{$[\mathrm{OII}] 7320+$} & - & - & 0.03 & 0.04 & 0.04 & 0.03 & 0.03 & 0.02 & 0.02 & - \\
\hline
\end{tabular}

obs $^{0}-$ obs $^{4}$ observations are described in Table 1 ;

Table 3. Modelling extinction corrected line ratios to $\mathrm{H} \beta$ from Guseva et al (2011)

\begin{tabular}{|c|c|c|c|c|c|c|}
\hline & obs $^{5}$ & $\bmod 5$ & obs $^{6}$ & $\bmod 6$ & $\mathrm{obs}^{7}$ & $\bmod 7$ \\
\hline [OII] 3728.8 & 0.86 & 086 & 0.98 & 0.99 & - & 1.03 \\
\hline [NeIII]3868+ & 0.38 & 0.40 & - & - & - & 0.49 \\
\hline $\mathrm{H} \gamma$ & 0.50 & 0.46 & - & - & 0.48 & 0.46 \\
\hline [OIII] 4363 & 0.09 & 0.10 & - & - & - & 0.07 \\
\hline HeI 4471.5 & 0.04 & 0.05 & - & - & - & 0.05 \\
\hline$[$ ArIV]4711 & 0.016 & 0.02 & $\ldots$ & - & - & 0.1 \\
\hline $\mathrm{H} \beta$ & 1 & 1 & 1 & 1 & 1 & 1 \\
\hline [OIII]5007+ & 9.78 & 9.20 & 5.89 & 5.60 & 10.20 & 10.30 \\
\hline HeI 5876 & 0.12 & 0.13 & - & - & 0.10 & 0.14 \\
\hline [OI] 6300 & 0.03 & 0.01 & 0.03 & 0.01 & - & 0.01 \\
\hline [SIII] 6312 & 0.02 & 0.02 & - & 0.02 & - & 0.01 \\
\hline [NII] $6548+$ & 0.18 & 0.17 & 0.13 & 0.17 & - & 0.17 \\
\hline $\mathrm{H} \alpha$ & 2.85 & 2.92 & - & 2.96 & - & 2.90 \\
\hline [SII] 6717 & 0.09 & 0.08 & 0.18 & 0.16 & 0.20 & 0.70 \\
\hline [SII] 6731 & 0.07 & 0.08 & - & - & 0.20 & 0.10 \\
\hline [AIII] 7136 & 0.06 & 0.05 & - & - & 0.08 & 0.06 \\
\hline [OII $] 7320+$ & 0.03 & 0.03 & - & - & - & 0.05 \\
\hline [SIII]9069 & 0.25 & 0.26 & - & - & 0.45 & 1.00 \\
\hline [SIV]10.61 & 0.47 & 0.60 & & - & - & 0.50 \\
\hline$[\mathrm{NeII}] 12.8$ & 0.04 & 0.04 & & - & - & 0.04 \\
\hline$[\mathrm{NeIII}] 15.56$ & 0.59 & 0.53 & - & - & - & 0.80 \\
\hline [SIII] 18.71 & 0.29 & 0.27 & - & - & - & 0.36 \\
\hline $\mathrm{H} \beta^{8}$ & & 0.07 & & 0.06 & - & 0.25 \\
\hline
\end{tabular}

obs $^{5}$ observations by Guseva et al and obs ${ }^{6}$ reported by Watson et al are described in Table 1; obs ${ }^{7}$ the broad line ratios presented by Guseva et al.; ${ }^{8} \mathrm{H} \beta$ absolute flux calculated at the nebula in $\mathrm{erg} \mathrm{cm}^{-2} \mathrm{~s}^{-1}$. 
Table 4. Model results

\begin{tabular}{lccccccccc}
\hline \hline & $\bmod 0$ & $\bmod 1$ & $\bmod 2$ & $\bmod 3$ & $\bmod 4$ & $\bmod 5$ & $\bmod 6$ & $\bmod 7$ & GIFH $^{7}$ \\
\hline $\mathrm{H} \beta{ }^{5}$ & 0.07 & 0.04 & 0.043 & 0.02 & 0.02 & 0.07 & 0.06 & 0.25 & - \\
$\mathrm{V}_{\mathrm{s}}\left(\mathrm{km} \mathrm{s}^{-1}\right)$ & 130 & 120 & 120 & 100 & 90 & 110 & 110 & 300 & - \\
$\mathrm{n}_{0}\left(\mathrm{n}_{0}\right)$ & 100 & 90 & 90 & 60 & 50 & 90 & 90 & 100 & - \\
$D(\mathrm{pc})$ & 2.93 & 0.44 & 2.93 & 1.03 & 1.71 & 4.30 & 4.00 & 1.40 & - \\
$\mathrm{T}_{*}\left(10^{4} \mathrm{~K}\right)$ & 7.40 & 7.40 & 7.40 & 7.20 & 7.20 & 6.50 & 5.50 & 6.50 & - \\
$\mathrm{U}-$ & 0.06 & 0.04 & 0.04 & 0.03 & 0.03 & 0.08 & 0.02 & 0.16 & - \\
$\mathrm{He} / \mathrm{H}$ & 0.1 & 0.1 & 0.1 & 0.1 & 0.1 & 0.1 & 0.1 & 0.1 & 0.1 \\
$\mathrm{~N} / \mathrm{H}^{6}$ & 0.14 & 0.14 & 0.14 & 0.12 & 0.12 & 0.14 & 0.14 & 0.14 & 0.12 \\
$\mathrm{O} / \mathrm{H}^{6}$ & 2.0 & 2.0 & 2.0 & 2.0 & 2.0 & 2.0 & 2.0 & 3.0 & 1.6 \\
$\mathrm{Ne} / \mathrm{H}^{6}$ & 0.4 & 0.6 & 0.4 & 0.6 & 0.6 & 0.3 & 0.4 & 0.3 & 0.3 \\
$\mathrm{~S} / \mathrm{H}^{6}$ & 0.05 & 0.04 & 0.05 & 0.06 & 0.05 & 0.07 & 0.08 & 0.08 & 0.04 \\
$\mathrm{Ar} / \mathrm{H}^{6}$ & 0.007 & 0.060 & 0.006 & 0.060 & 0.007 & 0.006 & 0.006 & 0.006 & 0.004 \\
\hline
\end{tabular}

\subsection{Line ratios from Prochaska et al (2004) and Margutti et al (2007) observations}

Margutti et al (2007) found that GRB031203 host is metal poor with $12+\log (\mathrm{O} / \mathrm{H})=8.12$. The GRB luminosity is low and the decay rate unusually slow, while underluminous GRB with fast decay rates at early times ( $t \leqslant 0.5$ days) have been suggested by Bloom et al (2003). In Tables 2 and 3 we compare model results with reddening-corrected line ratios in the next column. The observations by VLT presented by Prochaska et al (2004) in 2003 are followed in Table 2 by those of Margutti et al (2007, their table 4) at different epochs in 2003-2004. The fit is quite satisfying considering that the calculated line ratios are within the errors (20 percent for strong lines and 50 percent for weak lines). The observed line ratios constrain the models because $\mathrm{H} \alpha$ and $\mathrm{H} \beta$ are both observed in the spectra and oxygen is present by three ionization level lines. [OIII] $5007+/[\mathrm{OIII}] 4363$ line ratios are also available. It is known that they are low when the gas is heated to relatively high temperatures. This may occur when shocks are accounted for and photoionization is weak. Table 2 shows, on the contrary, that $[\mathrm{OIII}] 5007 /[\mathrm{OIII}] 4363$ are relatively high $(>80)$ in all the spectra, indicating that photoionization dominates. Sulphur appears by the $\mathrm{S}^{+}$and $\mathrm{S}^{++}$ions in the [SII]6717, 6731 doublet and in the [SIII] 6312 line, respectively. Although the [SIII]6312 line is generally weak and blended with the [OI]6300,6360 doublet, this line has been measured in the specific case of GRB031203, constraining the model.

The results of modelling are presented in Table 4 . The input parameter trends follow the small changes of the observed line ratios with time (Table 4 ). Those are within the observed errors and indicate that the photoionizing source of the host galaxy gas did not significantly vary during the observation period. A SB is generally adopted to explain the photoionizing flux in GRB hosts by the observer community. Our calculations confirm (see Guseva et al 2011) that a SB is adapted to explain GRB031203 host spectra. The ionization parameter decreases with the emitting gas distance from the hot source and/or because the radiation flux is prevented to reach the cloud by some obstructing matter. Accordingly, Table 4 shows a small decreasing trend of $U$ with time from December 2003 to September 2004, as the shock propagates throughout the host ISM. The SB effective temperature de- creases after September 2004. With regards to the shock parameters, we have found that shock velocities and preshock densities slightly decrease from December 2003 towards a minimum in September 2004. The geometrical thickness of the clouds, on the other hand, changes randomly due to fragmentation created by turbulence at the shock fronts.

All the line ratios observed in a single-time spectrum are reproduced by models implying that the radiation flux from the SB and the shock are coupled. Our models show $[\mathrm{SII}] 6717 /[\mathrm{SII}] 6731$ ratios $<1$ in some spectra, whereas the observed ones are $>1$, indicating very low densities of the emitting gas. Also the $[\mathrm{OI}] / \mathrm{H} \beta$ line ratios are underpredicted. We explain these results by the contribution of a large amount of ISM gas where the [OI] lines are strong and the densities are low.

In Figs. 3 and 4 the profiles of the electron temperature and electron density (top panel) and of the fractional abundances of the most significant ions (bottom panel) throughout a cloud are shown. Fig. 3 presents the case of the shock created by collision of the debris with the ISM clouds which contributes to reproduce most of the observed spectra $(\bmod 0-\bmod 6)$. The gas shows a maximum downstream temperature of $\sim 1.510^{5} \mathrm{~K}$ near the shock front $(\mathrm{T} \sim$ $\left.1.5 \times 10^{5}\left(\mathrm{~V}_{\mathrm{s}} / 100 \mathrm{~km} \mathrm{~s}^{-1}\right)^{2}\right)$. Therefore, the $\mathrm{O}^{++}$ion dominates throughout a large region of the cloud (Fig. 3). The density downstream which depends on the shock velocity is relatively low $\left(<10^{3} \mathrm{~cm}^{-3}\right)$. The gas downstream cools down following slow recombination because the density is low. The large region of gas at $\mathrm{T} \sim 10^{4} \mathrm{~K}$ explains why models accounting on pure photoionization reproduce the lines ratios as well as those accounting for the coupled effect of shock and photoionization. Fig. 3 shows that the gas is not fully recombined at a distance from the shock-front $\geqslant 1 \mathrm{pc}$ due to the low compression downstream which depends on $\mathrm{V}_{\mathrm{s}}$ and $\mathrm{n}_{0}$. It was found by modelling the line ratios that the emitting clouds are matter-bounded.

\subsection{Line ratios from Guseva et al (2011) and Watson et al (2011) observations}

Guseva et al presented VLT/X-shooter observations performed on March 2009. The same observations were discussed by Watson et al (2011) who added Spitzer spectroscopy of strong forbidden lines in the mid-IR in their ta- 

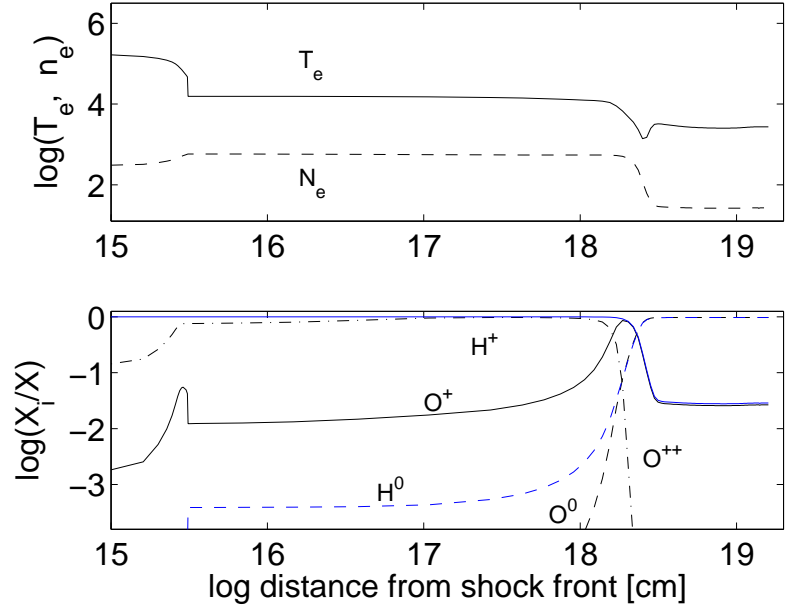

Figure 3. The shock front is on the left. Top panel: the distribution on $\mathrm{N}_{e}$ and $\mathrm{T}_{e}$ throughout the cloud calculated by model mod5. Bottom panel: the distribution of the $\mathrm{H}^{0} / \mathrm{H}, \mathrm{H}^{+} / \mathrm{H}, \mathrm{O}^{0} / \mathrm{O}$, $\mathrm{O}^{+} / \mathrm{O}$ and $\mathrm{O}^{++} / \mathrm{O}$ fractional abundance.
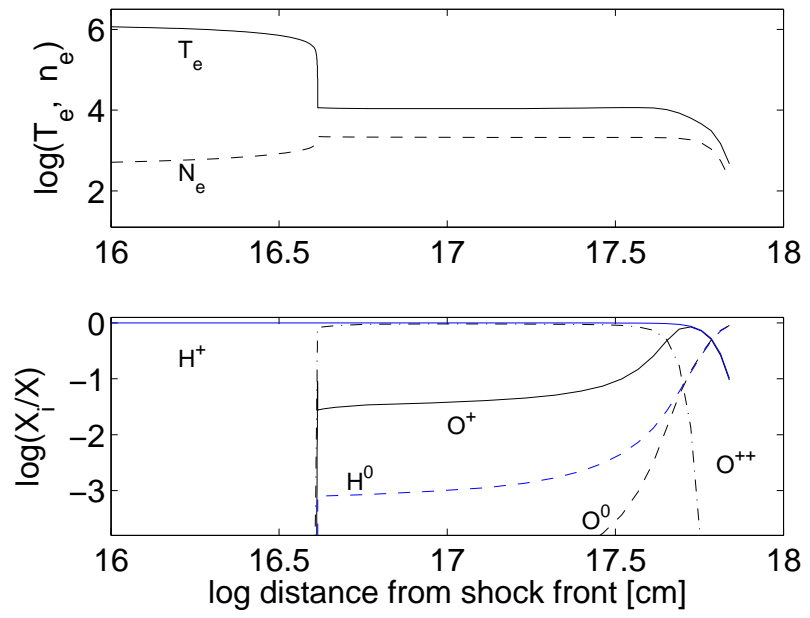

Figure 4. The same as in Fig. 3 for model mod7.

ble 3 . We compare model results to the reddening-corrected observed line ratios in Table 3.

The line ratios were previously modelled by Guseva et al adopting the code CLOUDY. They obtained very good results for all of them, therefore, they concluded that photoionization is the only source of the emitted lines. The fit is also good adopting a composite model (photoionization + shock). In fact, most of the lines observed are emitted from ions in the intermediate ionization levels II and III. Those lines are mainly produced by the photoionizing flux which heats the gas to $\leqslant 2-3 \times 10^{4} \mathrm{~K}$. Shocks can heat the gas to higher temperatures depending on the shock velocity, therefore shocks are mainly revaled by strong high ionization level lines and neutral lines (see Fig. 4). Moreover, a composite model can fit the observed SED from radio to $\mathrm{X}$-ray, because the bremsstrahlung maximum reaches relatively high frequencies depending on the shock velocity. The mutual heating and cooling between gas and dust grains lead to the bump in the IR calculated consistently with the line emission. Also, synchrotron radiation created by the Fermi mechanism at the shock front is often observed in the radio range.

The intensities of $\mathrm{H}$ recombination lines were calculated by Guseva et al by an electron temperature $\mathrm{T}_{\mathrm{e}}=12500 \mathrm{~K}$, and electron density $\mathrm{N}_{\mathrm{e}}=100 \mathrm{~cm}^{-3}$ and case B which indicates large optical depths. An oxygen metallicity $12+\log (\mathrm{O} / \mathrm{H})=8.20$ was evaluated by the $\mathrm{T}_{\mathrm{e}}$ method, in agreement with previous results (Prochaska et al 2004, Hammer et al 2006, etc). The element relative abundances calculated by Guseva et al (2011) are reported in the last column (GIFH) of Table 4 for comparison. The N/O abundance ratio results 0.075 . Oxygen metallicity is lower than that calculated by SUMA because the line ratios presented in Tables 2 and 3 are calculated integrating on the temperature decreasing gradient downstream. Chen (2018) suggested that satellite galaxies or tidal tails contain relatively more pristine gas around otherwise chemically evolved starforming galaxies (Thilker et al. 2009). This is true also for $\mathrm{N} / \mathrm{H}$.

However, the dynamical phenomena which follow the SB require the presence of shocks throughout the ISM host. Analysing the profiles of the strongest lines, Guseva et al disclosed that relatively high velocities $\left(\leqslant 400 \mathrm{~km} \mathrm{~s}^{-1}\right)$ contributed to the FWHM of the line profiles. They are seen as broad sockets in Guseva et al (2011) their fig. 7. The observed relatively large velocity distribution may be due to fragmentation following turbulence in the host ISM. Interestingly, complex gas kinematics were observed from the absorption lines in the GRB host environments of faint galaxies at $z \geqslant 1.5$ (Chen 2018) with a significant spread of velocities (e.g. Prochaska et al 2008). Chen (2018) claims that the relatively high velocity $\left(200-400 \mathrm{~km} \mathrm{~s}^{-1}\right)$ distribution in the foreground gas of the afterglows is "starburst driven outflows", most likely in the host galaxy. This scenario could produce shocks by collision with the ISM debris and clouds. Therefore we adopted composite models which account for shock and photoionization to explain the spectra. A blackbody radiation is used to represent the flux from the SB. We have modelled Guseva et al line ratios by the same type of models (mod5 and mod6) that were used for Margutti et al. spectra (Table 2). The broad line ratios are calculated by model $\bmod 7$ with a velocity $V_{\mathrm{s}}=300 \mathrm{~km} \mathrm{~s}^{-1}$, which is similar to the velocities yielding the sockets in the line profiles.

We assume that a relatively strong wind collides with the clouds on their internal edge facing the SB (Fig. 2, left panel). The shock front on the opposite (outer) edge is not significant because the cloud propagates in the outer ISM where the electron density is low. The shock front facing the SB is reached by the photoionization radiation (Fig. 2, left panel). The temperature $\left(\propto \mathrm{V}_{\mathrm{s}}{ }^{2}\right)$ in the downstream region close to the shock-front is relatively high, with a maximum $\mathrm{T}=1.35 \times 10^{6} \mathrm{~K}$. We report the calculated line ratios (model $\bmod 7)$ in Table 3 and the results of modelling in Table 4. The observed [SIII] $9069 / \mathrm{H} \beta$ line ratio is overestimated by model $\bmod 7$ by a factor $\sim 2$. The other mid-IR lines are satisfactorily reproduced.

The results calculated by the code SUMA depend on the stratification of the temperature and the density downstream which lead to the consistent fit of most of the line ratios. Fig. 4 shows the $\mathrm{T}_{\mathrm{e}}$ and $\mathrm{N}_{\mathrm{e}}$ distribution for model $\bmod 7$ which fits Guseva et al broad line observations. The 

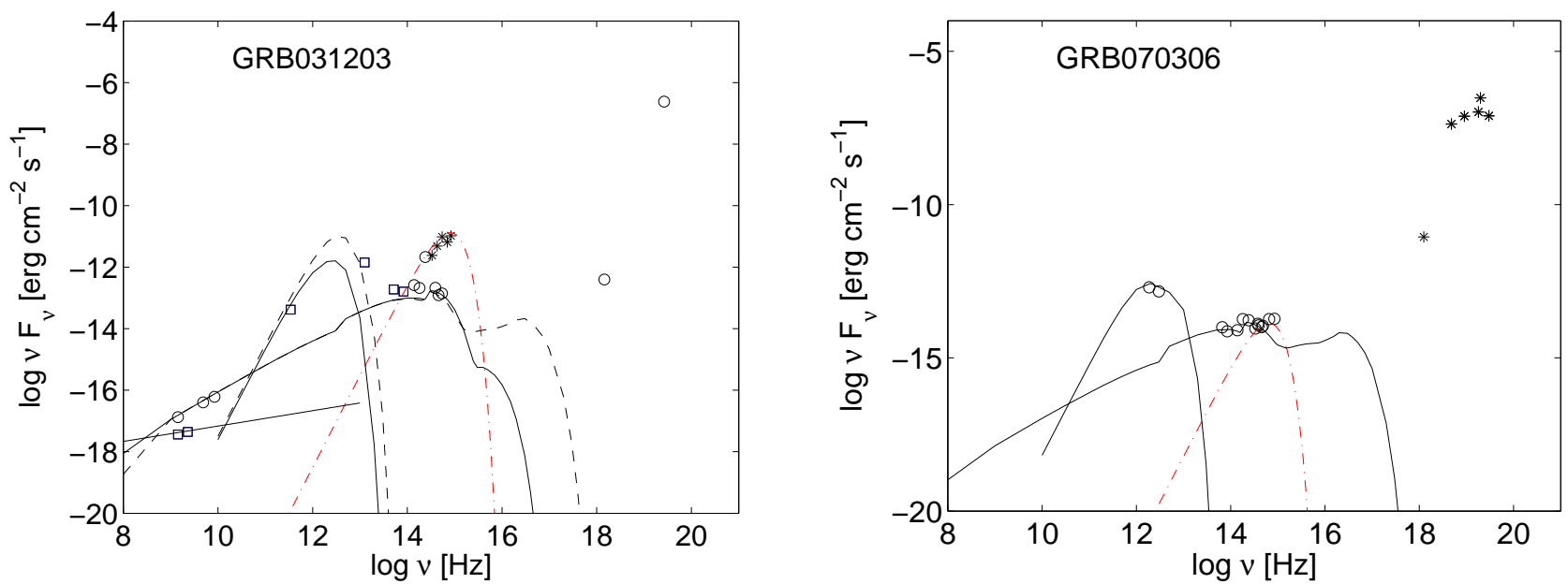

Figure 5. Left: SED of GRB031203. Black open circles in the X-ray: data from Firmani et al (2006) and Gendre et al (2006); black squares : data (VLA-Spitzer) from Watson et al; black asterisks: data from Margutti et al and Mazzali et al (2006); red dash-dotted line: bb flux calculated with $\mathrm{T}=10^{4} \mathrm{~K}$ representing the underlying old star population. Black solid lines: the bremsstrahlung and dust reradiation calculated by model mod1 (Table 4); black dashed lines: the bremsstrahlung and dust reradiation calculated by model mod7 (Table 4); black solid line in the radio: synchrotron radiation by the Fermi mechanism. Right: SED of LGRB070306 adapted from Contini (2018a).

gas cools down slowly near the shock front because recombination coefficients are small for $\mathrm{T} \geqslant 10^{5} \mathrm{~K}$. Then, following the strong cooling rate due to UV and optical line emission, the temperature drops to $\leqslant 10^{4} \mathrm{~K}$. The secondary diffuse radiation maintains the gas at $\geqslant 10^{4} \mathrm{~K}$ throughout a large region. The gas recombines at a distance from the shock-front $\leqslant 1$ pc. The model which best reproduces the data is radiationbounded (Table 4). In the bottom diagram of Fig. 4 the distribution of the fractional abundance of $\mathrm{H}^{0} / \mathrm{H}, \mathrm{H}^{+} / \mathrm{H}$, $\mathrm{O}^{0} / \mathrm{O}, \mathrm{O}^{+} / \mathrm{O}$ and $\mathrm{O}^{++} / \mathrm{O}$ ions is shown.

\section{CONTINUUM SED}

Spitzer photometric data for GRB031203 were observed by Watson et al (2011) by IRAC. Radio continuum observations were obtained on January 2008 using ATCA (Watson et al 2011, table 2). In Fig. 5 (left panel) we present the modelling of GRB031203 continuum SED on the basis of Watson et al data. To reproduce the data, we use the bremsstrahlung calculated by the models that were selected by fitting the line ratios. The continua calculated by models $\bmod 1$ and $\bmod 7$ are selected because the former represents emission at earlier times and the latter represents the broad line component which appears later in the line profiles. For each model two curves are shown in the figure, one is gas bremsstrahlung which covers the frequency range from radio to soft X-rays, the other in the IR is reprocessed radiation by dust consistently calculated. The bremsstrahlung calculated by the models shows similar trends in the radiooptical range. It reproduces the GRB031203 data in the radio and defines the bottom limit to the IR data which are generally nested within a black-body curve. This bump is explained by the flux emitted from the underlying old star population. For GRB031203 the old star best fitting temperature is $10^{4} \mathrm{~K}$. The continuum calculated by model $\bmod 7$ reaches higher frequencies than mod1 because the high velocity shock heats the gas to higher temperatures. Synchrotron radiation in the radio range, created by the Fermi mechanism at the shock front, is most likely fitted by mod7. Thermal bremsstrahlung at $\nu<1 \mathrm{GHz}$ shows self absorption by high density. In the right panel of Fig. 5 we report the SED of LGRB070306 (Contini 2018a, fig.1) for comparison. The underlying old star contribution hardly emerges from the LGRB070306 bremsstrahlung. The LGRB031203 two data in the X-ray range (Firmani et al 2006, Gendre et al 2006) cannot be reproduced by the models presented in Table 4 because they are emitted from the afterglow. In the GRB070306 SED (Fig. 5 right panel) and for other LGRB reported by Contini (2018a), various data in the X-ray appear at higher frequencies. Only a few of them are observed in GRB031203. In fact, they represent the afterglow which is weak in GRB031203.

\section{CONCLUDING REMARKS}

The spectra observed from the long GRB031203 ( $\mathrm{z}=0.1055)$ host galaxy are emitted from clouds moving with different velocities throughout the ISM. It was explained by Chen (2018) that the distribution of velocities depends on SB driven outflows. The lines observed by Guseva et al in March 2009 show FWHM profiles of $\sim 100 \mathrm{~km} \mathrm{~s}^{-1}$, similar to those observed in 2003-2004 by Margutti et al, but with a FWHM $\sim 300 \mathrm{~km} \mathrm{~s}^{-1}$ component. We have reproduced the observed line ratios at different times by two main models. We suggest that the spectra observed at early times by Margutti et al are emitted downstream of head-on shock created by collision of the SB ejected debris with nearly standing ISM clouds. The shock velocity is mainly determined by the SB debris. The spectra corresponding to FWHM of $\sim 300 \mathrm{~km} \mathrm{~s}^{-1}$ are emitted downstream of the head-on-back shock created by collision of the fast wind from SB stars with the ejected debris. 
The results obtained by the detailed modelling of LGRB031203 host line ratios observed at different times are presented in Table 4 . The models account for the coupled effects of shocks and photoionization from the SB. The line ratios are enough to constrain the models because $\mathrm{H} \alpha$, $\mathrm{H} \beta$ and oxygen in three different ionization levels appear in the spectra. The [OIII] $5007+/[\mathrm{OIII}] 4363$ line ratios are available. Sulphur is present by the $\mathrm{S}^{+}$and $\mathrm{S}^{++}$ions as the [SII]6717, 6731 doublet and the [SIII] 6312 line. Although the [SIII]6312 line is generally weak and blended with the [OI]6300,6363 doublet, this line is measured in the specific case of GRB031203. The results show that the main physical parameters $\left(\mathrm{V}_{\mathrm{s}}, \mathrm{n}_{0}\right.$ and the ionization parameter) show a small decreasing trend from December 2003 to a minimum in September 2004. The SB effective temperature decreases from September 2004 to March 2009. The element abundances relative to $\mathrm{H}$ are calculated at each epoch. $\mathrm{S} / \mathrm{H}$ slightly decreases after September 2004. The O/H ratio is found higher in the high velocity clouds than in the low ones by a factor of 1.5, but it is still lower than solar by a factor of $\sim 2$. The comparison of the results obtained by the detailed modelling of the spectra with the results obtained using the $\mathrm{T}_{\mathrm{e}}$ method by the observers shows that oxygen is less depleted in GRB0031203 host than predicted by previous analysis. Prochaska et al (2004) and Guseva et al (2011) found $\log (\mathrm{O} / \mathrm{H})+12=8.2$, Margutti et al (2008) estimated 8.12. We found 8.3. In Fig. 6 we report N/O results for LGRB hosts, SB, low-luminosity nearby galaxies and HII regions in local galaxies (see Table 5) as a function of $\mathrm{z}$. It was found that N/O in LGRB hosts follows a decreasing trend with decreasing z, while N/O for supernovae (SN), SGRB hosts and AGN increases with decreasing z (Contini 2017, 2019). Contini (2017) suggested that N/O ratios in SN hosts increase due to secondary $\mathrm{N}$ production towards low z (0.01). They accompany the growing trend of AGN and low-ionization nuclear emission-line regions (LINER)(Contini 2018b). N/O ratios in LGRB hosts decrease rapidly between $\mathrm{z}>1$ and $\mathrm{z} \sim 0.1$ following the $\mathrm{N} / \mathrm{H}$ trend and reach the characteristic N/O ratios calculated for the HII regions in local and nearby galaxies. The location of the $\mathrm{N} / \mathrm{O}$ ratio calculated for the LGRB031203 host in Fig. 6 diagram reveals that $\mathrm{O} / \mathrm{H}$ is relatively low. The low $[\mathrm{NII}] / \mathrm{H} \beta$ line ratios in the Margutti et al spectra confirm that $\mathrm{N} / \mathrm{H}$ is low in LGRB at low z. We have found that the low $\mathrm{O} / \mathrm{H}$ and $\mathrm{N} / \mathrm{H}$ abundance ratios in the GRB031203 host are accompanied by low Ne/H, S/H and $\mathrm{Ar} / \mathrm{H}$. O, N, Ne, S and Ar are among the most abundant heavy elements. Moreover, the fit of the calculated $\mathrm{He}$ lines could improve adopting $\mathrm{He} / \mathrm{H}=0.08$ (Tables 2 and 3 ) in agreement with Guseva et al (2011). We will be able to confirm whether more elements are depleted in LGRB host galaxies when $\mathrm{C}, \mathrm{Si}, \mathrm{Mg}$, etc lines which are strong in the $\mathrm{UV}$ and in the IR will be available from the observations.

Modelling the SED of the GRB031203 host, we have found that the underlying old star population emerges from the bremsstrahlung by a black-body bump in the IR corresponding to a temperature of $10^{4} \mathrm{~K}$. The thermal bremsstrahlung and the synchrotron radiation created by the Fermi mechanism at the shock front which appear in the SED radio range, belong to different observations.

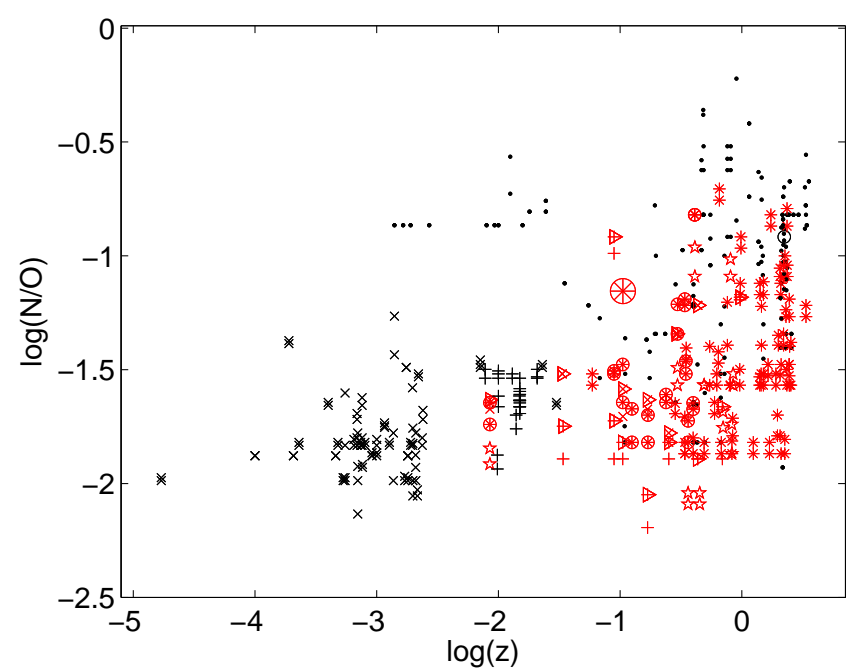

Figure 6. N/O abundance ratio results as function of the redshift. Large encircled red asterisk: N/O calculated for GRB031203. Other symbols are described in Table 5.

Table 5. Symbols in Fig. 6

\begin{tabular}{llc}
\hline \hline symbol & object & Ref. \\
\hline red asterisks & LGRB hosts & $(1)$ \\
red triangle+cross & LGRB hosts & $(2)$ \\
red pentagrams & LGRB hosts & $(3)$ \\
red triangle +plus & LGRB hosts with WR stars & $(4)$ \\
red encircled asterisks & LGRB at low z & $(5)$ \\
black dots & star-burst galaxies & $(6)$ \\
black plus & low-luminosity nearby galaxies & $(7)$ \\
black cross & HII regions in local galaxies & $(8)$ \\
\hline
\end{tabular}

(1) Krühler et al. (2015); (2) Savaglio et al. (2009); (3) Contini (2016, table 8); (4) Han et al.(2010); (5) Niino et al.(2016); (6) reported by Contini (2014) and references therein; (7) Marino et al. (2013); (8) Berg et al. (2012);

\section{ACKNOWLEDGEMENTS}

I am grateful to the referee for many helpful remarks.

\section{REFERENCES}

Aldrovandi, S.M.V. \& Péquignot, D. 1973 A\&A, 25, 137

Bell, A.R. 1977, MNRAS, 179, 573

Berg, D.A. et al 2012, ApJ, 754, 98

Berger, E. 2009, ApJ, 690, 231

Bloom, J.S., Frail, D.A., Kulkarni, S.R. 2003, ApJ, 591, L13

Chen, H-W. 2018, MNRAS arXiv:1110.0487

Contini, M. 2019, submitted

Contini, M. 2018a, arXiv:1801.03312

Contini, M. 2018b, A\&A, 620, 37

Contini, M. 2017, MNRAS, 469, 3125

Contini, M. 2016, MNRAS, 460, 3232

Contini, M. 2014, A\&A, 564, 19

Contini, M. \& Viegas-Aldrovandi, S.M. 1990 ApJ, 350, 125

Contini, M. \& Aldrovandi, S.M.V. 1983 A\&A, 127, 15

Contini, M. \& Shaviv, G. 1980, A\&A, 88, 117 
Cox, D.P. 1972, ApJ, 178, 143

Cox, D.P. \& Tucker, W.H. 1969, ApJ, 157, 1157

Gendre, B., Corsi, A., Piro, L. 2006, A\&A, 455, 803

Crowther, P.A., Dessart, L., Hillier, D.J., Abbott, J.B., Fullertone, A.W. 2002, A\&A, 392, 653

Giannios, D., Mimica, P., Aloy, M.A. 2008, A\&A, 478, 747

Gotz, D., Meneghetti, S.,Beck, M., Borkowski, J. 2003, GCN Circ. 2459

Gotz, D. Freyer, C.L., Woosley, S.E., Langer, N., Hartmann, D.H. 2003, ApJ, 591, 288

Guseva, N.G., Izotov, Y.I., Fricke, K.J., Henkel, C. 2011, A\&A, 534, A84

Firmani, C., Ghisellini, G., Avila Reese, V., Ghirlanda, G. 2006, MNRAS, 370, 185

Fruchter, A.S. et al 2006, Nature, 441, 463

Hammer, F., Flores, H., Schaerer, D. et al 2006, A\&A, 454, 103

Han, X. H., Hammer, F., Liang, Y. C., Flores, H., Rodrigues, M., Hou, J. L., Wei, J. Y. 2010, A\&A, 514, 24

Heger, A., Woosley, S.E., Langer, N., Spruit, H.C. 2004, in Stellar rotation, IAU Symp., 215, 591

Hirschi, R., Meynet, G., Maeder, A. 2005, A\&A, 443, 581

Hjorth, J.J. 2003, Nature, 423, 847

Hsia, C.H., Lin, H.C., Huang, K.J., Urata, Y., Ip, W.H., Tamagawa, T. 2003, GCN Circ. 2470

Kobayashi, S. \& Zhang, B. 2003, ApJ, 597, 455.

Kouveliotou, C., Meegan, C.A., Fishman, G.J et al 1993, ApJ, 413, L101

Krühler, T. et al 2015 A\&A, 581, 125

MacFadyen, A.I. \& Woosley, S.E. 1999, ApJ, 524, 262

Maeder, A, Meynet, G., Hirschi, R. 2005, ASPC, 336, 79

Margutti, R. et al 2008, A\&A, 474, 815

Marino, R.A. et al 2013, A\&A, 229, 114

Mazzali, P.A. et al 2006, ApJ, 645, 1323

Meneghetti, S. \& Gotz, D. 2003, GCN Circ.2460

Niino, Y. et al 2016 Publ. Astron. Soc. Japan, arXiv:1616.01983

Perley, D.A. , Niino, Y., Tanvir, N.R., Vergani, S.D., Fynbo, J.P.U. 2016, ApJ arXiv:1602.00770

Piran, T. 2004 RvMP, 76, 1143 (arXiv:astro-ph/0405503)

Prochaska, J.X. et al 2004, ApJ, 611, 200

Sari, R.\& Piran, T. 1999, ApJ, 520, 641

Savaglio, S., Glazerbrook, K., Le Borgne, D. 2009, ApJ, 691, 182

Schiano, A.V.R. 1985, ApJ, 299, 24

Schiano, A.V.R. 1996, ApJ, 302, 81

Tagliaferri, Covino, S., Fugazza, D., Chincarini, G., Malesani, D., Della Valle, M., Stella, L. 2004IAUC.8308

Thilker, D.A. et al. 2009, Nature, 457, 990

Viegas-Aldrovandi, S.M. \& Contini, M. 1989, A\&A, 215, 253

Viegas, S.M. \& Contini, M. 1994, ApJ, 428, 113

Vink, J.S., de Koter, A. 2005, A\&A, 442, 587

Vogel, M. \& Nussbaumer, H. 1994, A\&A, 284, 145

Watson, D. et al 2011, ApJ, 741, 58

Wei, J.-J., Wu, X.-F., Melia, F., Wei, D.-M., Feng, L.-L. 2014, MNRAS, 439, 3329

Williams, R.E. 1973, MNRAS, 164, 411

Williams, R.E. 1967, ApJ, 147, 556

Wolf, C. \& Podsiadlowski, P. 2007, MNRAS, 375, 1049

Woosley, S.E. 1993, ApJ, 405, 237

Woosley, S.E. \& Bloom, J. S. 2006, ARA\&A, 44, 507

Zhang, B. \& Kobayashi, S. 2005, ApJ, 628, 315 